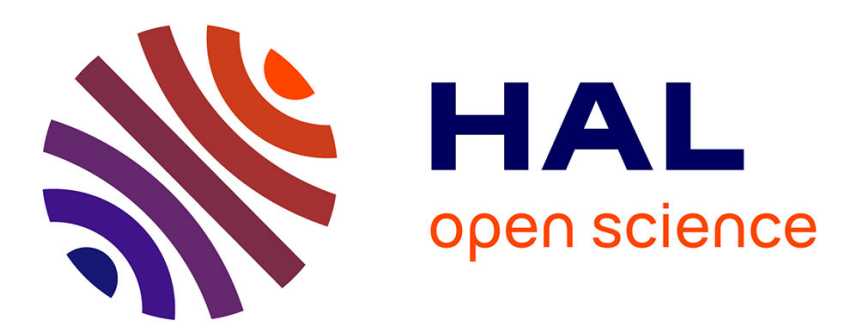

\title{
High order sliding mode control for sensorless trajectory tracking of a PMSM
}

Romain Delpoux, Thierry Floquet

\section{To cite this version:}

Romain Delpoux, Thierry Floquet. High order sliding mode control for sensorless trajectory tracking of a PMSM. International Journal of Control, 2014, pp.2140-2155. 10.1080/00207179.2014.903563 . hal-00957787

\section{HAL Id: hal-00957787 https://hal.science/hal-00957787}

Submitted on 11 Mar 2014

HAL is a multi-disciplinary open access archive for the deposit and dissemination of scientific research documents, whether they are published or not. The documents may come from teaching and research institutions in France or abroad, or from public or private research centers.
L'archive ouverte pluridisciplinaire HAL, est destinée au dépôt et à la diffusion de documents scientifiques de niveau recherche, publiés ou non, émanant des établissements d'enseignement et de recherche français ou étrangers, des laboratoires publics ou privés. 


\title{
High order sliding mode control for sensorless trajectory tracking of a PMSM
}

\author{
R. Delpoux ${ }^{a, b *}$ and T. Floquet ${ }^{a, b}$ \\ ${ }^{a}$ LAGIS UMR CNRS 8219, École Centrale de Lille, 59651 Villeneuve d'Ascq Cedex, France; \\ ${ }^{b}$ Team Non-A, Inria Lille Nord Europe, France
}

(v1.2 released Septembre 2013)

\begin{abstract}
The paper presents a new sensorless approach for permanent magnet synchronous motor (PMSM). Current sensors are assumed available, but position and velocity sensors are not. Based on the electrical equations, sliding mode observers are designed to estimate the back-EMF of the motor. These estimations are used to reconstruct the position and the velocity. From this estimation, a robust sliding mode control is developed which ensures the position tracking of the motor. A new reference frame is used that presents advantages similar to the standard $(d-q)$ frame, but without the need for a position sensor. The efficiency of the algorithm is shown through experimental results. The approach is potentially applicable to other types of synchronous motors as well.
\end{abstract}

Keywords: PMSM, sensorless, sliding modes, reference rotating frame.

\section{Introduction}

Permanent Magnet Stepper Motors (PMSM's) are widely used in industry for position control, especially in manufacturing applications. PMSM's are more robust than brush DC motors and produce high torque per volume. They are often controlled in open-loop, although the potential loss of synchronism limits operation away from resonances and from high acceleration trajectories. Using closed-loop control methods with position sensors of sufficient accuracy can solve these problems. Recent research has focussed on whether the performance of closed-loop control methods could be achieved using sensorless systems. In this case, sensorless refers to systems that do not have position sensor nor velocity, although current sensors are still assumed to be available.

One can find work in the literature which deals with the design of a control supposing that the rotor position is known (Chiasson and Novotnak 1993, Nollet et al. 2008, Defoort et al. 2009). Concerning "sensorless" based control, different approaches have been treated. One can refer to the overviews of (Johnson et al. 1999, Schroedl 2004) treating brushless DC motor and PMSM, respectively. Among the different approaches to treat this problem, the most current are the high frequency injection method (Jang et al. 2004, Zhu et al. 2009), observer based approach such as Extended Kalman Filter (Bolognani et al. 2001, Bendjedia et al. 2012), linear observer (Son et al. 2002), nonlinear observer (Ortega et al. 2011, Shah et al. 2011, Khlaief et al. 2011, Tomei and Verrelli 2011), adaptive interconnected observer (Hamida et al. 2013) and sliding mode observers (Kim et al. 2011, Fiter et al. 2010, Zribi et al. 2001). Although not exhaustive, this list gives an idea on the issues.

This article presents a solution for mechanical sensorless control of a PMSM based on high order sliding modes. Sliding mode theory is commonly used for the design of robust nonlinear observers or control laws. Indeed, sliding modes provide very good properties with respect to

* Corresponding author. Email: romain.delpoux@ec-lille.fr 
perturbations and uncertainties. Another interesting property of the sliding modes is the finite time convergence, which unlike the asymptotic convergence, do not requires the use of separation principle theorem to prove the convergence of an observer based control. The use of second order sliding modes reduces the high frequency commutations known as Chattering. In the literature, a wide range of applications using sliding modes can be found : (Bartolini et al. 2003, Bartolini and Pisano 2003, Butt and Bhatti 2008, Canale et al. 2008, Davila et al. 2009, Defoort et al. 2008, Drakunov et al. 2005, Floquet et al. 2003, Fridman et al. 2007, Martinez et al. 2008, Pisano et al. 2008, Riachy et al. 2008).

From a control engineer perspective, the most used control laws are designed in the field oriented frame, referred as $d-q$ frame. It offers several advantages since it provides a simplified structure for the control, by avoiding sinusoidal functions. This frame from a sensorless point of view is not usable since it relies on the use of the position sensor. The proposed approach is based on the use of a different reference frame which is obtained from a reference position instead of a measured position. This frame permits to have the same properties than the $d-q$ frame, with the advantage that it does not require the actual motor position. In the literature, this frame was introduced for sensorless control and parameter identification in (Morimoto et al. 2002, 2006, Zheng et al. 2007, Shi et al. 2012, Delpoux et al. 2012).

The main contribution of the article is the use of second order sliding modes observers, based on Super Twisting Algorithms (STA) for the estimation of the position and the velocity of the motor. The observers use the input voltages and the measured currents only, to estimate the motor back-EMF. From this estimation, both position and velocity can be reconstructed. These estimations are used for the control. Here, we propose an observer based sliding mode control for the motor position trajectory tracking. This control is based on the flatness property of the PMSM (Sira-Ramírez 2000). The theory is validated through experiments that were performed using a test bench available at the LAGIS laboratory at the École Centrale de Lille.

This article is divided into four parts. The first section presents the model of the PMSM in the three different frames, particularly the reference rotating frame. The higher order sliding modes as well as the control strategy are also presented in this section. Then second order sliding mode observers are developed to estimate the position, velocity and acceleration of the PMSM in the section 3. The control law is derived Section 4 together with the stability of the observer based control. The last section is devoted to experimental results.

\section{Problem Statement}

\section{$2.1 \quad$ PMSM Model}

The model of the PMSM is given in three different frames (see Fig. 1). After a description of the model in the variables $(a-b)$, the model in the rotating frame $(d-q)$ is presented. This model is useful for the control law design. However this model is obtained from the measured position. In sensorless application, the use of this model is not suitable. Hence a reference rotational frame based on the reference trajectory, called $(f-g)$ frame is introduced.

\subsubsection{Model in the phase variables $(a-b)$}

Equations (1) give the standard PMSM model in the phase (or winding) variables

$$
\left\{\begin{array}{l}
L \frac{d i_{a}}{d t}=v_{a}-R i_{a}+K \omega \sin \left(n_{p} \theta\right), \\
L \frac{d i_{b}}{d t}=v_{b}-R i_{b}-K \omega \cos \left(n_{p} \theta\right), \\
J \frac{d \omega}{d t}=K\left(i_{b} \cos \left(n_{p} \theta\right)-i_{a} \sin \left(n_{p} \theta\right)\right)-f_{v} \omega-C_{r}, \\
\frac{d \theta}{d t}=\omega,
\end{array}\right.
$$




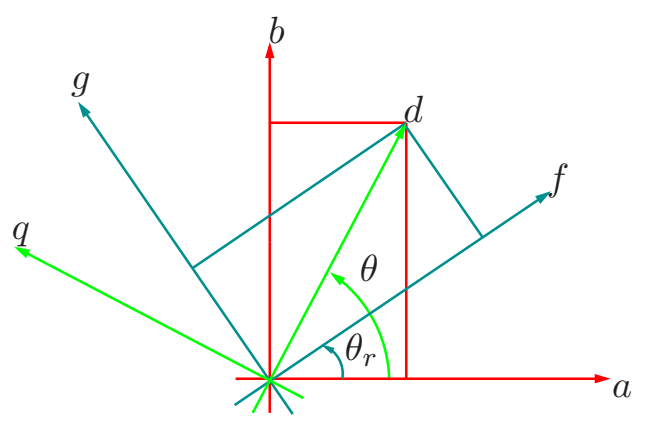

Figure 1. Variables in the different frames.

where $v_{a}$ and $v_{b}$ are the voltages applied to the two phases of the PMSM, $i_{a}$ and $i_{b}$ are the two phase currents, $L$ is the inductance of a phase winding, $R$ is the resistance of a phase winding, $K$ is the back-EMF constant, $\theta$ is the angular position of the rotor, $\omega$ is the angular velocity of the rotor, $n_{p}$ is the number of pole pairs (or rotor teeth), $J$ is the moment of inertia of the rotor (including the load), $f_{v}$ is the coefficient of viscous friction and $C_{r}$ is the load torque, which may vary as a function of the time.

\subsubsection{Model in the rotating frame $(d-q)$}

The phase model can be transformed using Park's transformation (Park 1929):

$$
\begin{aligned}
{\left[i_{d}, i_{q}\right]^{T} } & =U(\theta)\left[i_{a}, i_{b}\right]^{T}, \\
{\left[v_{d}, v_{q}\right]^{T} } & =U(\theta)\left[v_{a}, v_{b}\right]^{T},
\end{aligned}
$$

where

$$
U(\theta)=\left[\begin{array}{cc}
\cos \left(n_{p} \theta\right) & \sin \left(n_{p} \theta\right) \\
-\sin \left(n_{p} \theta\right) & \cos \left(n_{p} \theta\right)
\end{array}\right] .
$$

Using this change of coordinates, the system (1) is transformed into the so-called $(d-q)$ model

$$
\left\{\begin{array}{l}
L \frac{d i_{d}}{d t}=v_{d}-R i_{d}+n_{p} L \omega i_{q} \\
L \frac{d i_{q}}{d t}=v_{q}-R i_{q}-n_{p} L \omega i_{d}-K \omega \\
J \frac{d \omega}{d t}=K i_{q}-f_{v} \omega-C_{r} \\
\frac{d \theta}{d t}=\omega
\end{array}\right.
$$

The $(d-q)$ transformation is commonly used for PMSM's (and synchronous motors in general), because it results in constant voltages and currents at constant speed (instead of the highfrequency phase variables). Also, the model highlights the role of the quadrature current $i_{q}$ in determining the torque. However, the $(d-q)$ transformation is based on the position $\theta$, which is not directly available in sensorless applications.

\subsubsection{Model in the rotating reference frame $(f-g)$}

To overcome the problems caused by the use of the position in the $d-q$ frame, a different reference frame that uses a reference position instead of the real position is proposed in this 
work. The transformation is expressed in matrix form as

$$
\begin{aligned}
{\left[i_{f}, i_{g}\right]^{T} } & =U\left(\theta_{r}\right)\left[i_{a}, i_{b}\right]^{T}, \\
{\left[v_{f}, v_{g}\right]^{T} } & =U\left(\theta_{r}\right)\left[v_{a}, v_{b}\right]^{T},
\end{aligned}
$$

where

$$
U\left(\theta_{r}\right)=\left[\begin{array}{cc}
\cos \left(n_{p} \theta_{r}\right) & \sin \left(n_{p} \theta_{r}\right) \\
-\sin \left(n_{p} \theta_{r}\right) & \cos \left(n_{p} \theta_{r}\right)
\end{array}\right] .
$$

and $\theta_{r}$ is an arbitrary reference position.

The PMSM model in the transformed variables is

$$
\left\{\begin{aligned}
L \frac{d i_{f}}{d t} & =v_{f}-R i_{f}-K \omega \sin \left(n_{p} \Delta \theta\right)+L n_{p} \omega_{r} i_{g} \\
L \frac{d i_{g}}{d t} & =v_{g}-R i_{g}-K \omega \cos \left(n_{p} \Delta \theta\right)-L n_{p} \omega_{r} i_{f}, \\
J \frac{d \omega}{d t} & =K\left(i_{f} \sin \left(n_{p} \Delta \theta\right)+i_{g} \cos \left(n_{p} \Delta \theta\right)\right)-f_{v} \omega-C_{r}, \\
\frac{d \theta}{d t} & =\omega
\end{aligned}\right.
$$

where $\Delta \theta=\theta-\theta_{r}$ and $\omega_{r}=d \theta_{r} / d t$. The $(f-g)$ frame is potentially useful as $\theta_{r}$ may be defined as the reference position that the motor is supposed to track. Then, the $(f-g)$ model approximates the $(d-q)$ model, with the advantage that it is valid and computable even if $\theta_{r}$ is not exactly equal to $\theta$ which cannot be used in the control law.

\subsection{High order Sliding mode}

The principle of higher order sliding mode control is to constrain the system trajectories to reach and stay, after a finite time, on a given sliding manifold $\mathcal{S}_{r}$ in the state space (Emel'yanov et al. 1986, Perruquetti and Barbot 2002). Consider a system whose dynamics is given by:

$$
x=f(t, x)+g(t, x) u,
$$

where $x \in \mathrm{R}^{n}$ is the system state, $u \in \mathrm{R}$ is the control and $f, g$ are sufficiently smooth vector fields. The sliding manifold is defined by the vanishing of a corresponding sliding variable $\mathcal{S}$ : $\mathrm{R}^{+} \times \mathrm{R}^{n} \rightarrow \mathrm{R}$ and its successive time derivatives up to a certain order. In the literature, the "sliding" algorithms are mainly of second order. The second order sliding set is written:

$$
\mathcal{S}_{2}=\left\{(t, x) \in \mathrm{R}^{+} \times \mathrm{R}^{n}: S(t, x)=\dot{S}(t, x)=0\right\} .
$$

The second derivative w.r.t the time of the sliding surface can be written:

$$
\ddot{S}=\phi(t, S, \dot{S})+\varphi(t, S, \dot{S}) U
$$

where $U=u$ (respectively $U=\dot{u}$ ) for systems of relative degree 2 (relative degree 1 ) w.r.t $S$.

Under the assumption that there exists positive constants $S_{0}, k_{m}, K_{M}, C_{0}$ such that $\forall x \in \mathrm{R}^{n}$ 
and $|S(t, x)|<S_{0}$, the system satisfies the following conditions:

$$
\left\{\begin{array}{l}
0<k_{m} \leq \mid \varphi\left(t, S, \dot{S} \mid \leq K_{M}\right. \\
|\phi(t, S, \dot{S})|<C_{0}
\end{array}\right.
$$

Different sliding mode algorithms can be found in the literature, here are recalled only the algorithms used in the article: the Super Twisting Algorithm and the Twisting Algorithm.

\subsubsection{Super Twisting Algorithm}

For systems of relative degree 1, the Super Twisting Algorithm will be used (Levant 1993, 2001). It is a second order sliding mode defined by:

$$
u_{s t}(S)=u_{1}(S)+u_{2}(S)
$$

with:

$$
\left\{\begin{array}{l}
\dot{u}_{1}(S)=-\alpha \operatorname{sgn}(S) \\
u_{2}(S)=-\lambda|S|^{\frac{1}{2}} \operatorname{sgn}(S)
\end{array}\right.
$$

The finite time stability of the algorithm is proved using a Lyapunov function. With relative degree equal to 1 , one has:

$$
\dot{S}=u_{s t}+\Pi \text {. }
$$

where $\Pi$ is a bounded perturbation, whose time derivative is also bounded. From the control law defined equation (13), this equation can be written:

$$
\begin{aligned}
\dot{S} & =\xi-\lambda|S|^{1 / 2} \operatorname{sgn}(S), \\
\dot{\xi} & =\Pi-\alpha \operatorname{sgn}(S),
\end{aligned}
$$

where $\alpha$ and $\lambda$ are positives gains to be defined. The finite time stability of the algorithm is derived from (Barbot and Floquet 2010).

Consider the equations of the system (16) and note : $\psi=\left[\begin{array}{l}\psi_{1} \\ \psi_{2}\end{array}\right]=\left[\begin{array}{c}|S|^{1 / 2} \operatorname{sgn}(S) \\ \xi\end{array}\right]$.

Leading to:

$$
\begin{aligned}
\dot{\psi} & =\left|\psi_{1}\right|^{-1}\left[\begin{array}{ll}
-\lambda & 1 \\
-\alpha & 0
\end{array}\right] \psi+\left[\begin{array}{c}
0 \\
\Pi
\end{array}\right] \\
& =\left|\psi_{1}\right|^{-1}\left(M \psi+\left[\begin{array}{c}
0 \\
\left|\psi_{1}\right| \Pi
\end{array}\right]\right) .
\end{aligned}
$$

Define the Lyapunov candidate function:

$$
V=\psi^{T} P \psi
$$

where $P=\left[\begin{array}{ll}p_{1} & p_{3} \\ p_{3} & p_{2}\end{array}\right]$ is a symmetric positive definite matrix. The time derivative of $V$ along the solution of (17) is given by:

$$
\dot{V}=\left|\psi_{1}\right|^{-1}\left(\psi^{T}\left(M^{T} P+P M\right) \psi+2 \psi^{T} P\left[\begin{array}{c}
0 \\
\left|\psi_{1}\right| \Pi
\end{array}\right]\right) .
$$


where:

$$
2 \psi^{T} P\left[\begin{array}{c}
0 \\
\left|\psi_{1}\right| \Pi
\end{array}\right] \leq k_{1} \psi_{1}^{2}+k_{2} \psi_{2}^{2},
$$

with $k_{1}=\Pi\left(2\left|p_{3}\right|+\epsilon\right)$ and $k_{2}=\Pi \frac{p_{2}^{2}}{\epsilon}$, for all $\epsilon>0$.

Thus:

$$
\dot{V} \leq\left|\psi_{1}\right|^{-1} \psi^{T}\left(M^{T} P+P M+\left[\begin{array}{cc}
k_{1} & 0 \\
0 & k_{2}
\end{array}\right]\right) \psi .
$$

Because $M$ is a Hurwitz matrix, the observer gains $\alpha$ and $\lambda$ can be chosen such that the matrix

$$
-Q=M^{T} P+P M+\left[\begin{array}{cc}
k_{1} & 0 \\
0 & k_{2}
\end{array}\right],
$$

is negative definite. By application of LaSalle theorem, the finite time convergence $\psi$ toward zero is proven (Barbot and Floquet 2010), i.e. the finite time convergence of $S$ and $\xi$ toward zero. Thus after finite time $\xi=0$ leading to $\dot{S}=0$.

In some cases, more particularly the observers, we resort a linear stabilizing term. This additive term aims to reduce the noise and accelerating the convergence towards the sliding surface. The approach given in (Shen and Huang 2009) extends the preceding results to this case.

\subsubsection{Twisting Algorithm}

For systems of relative degree 2, the Twisting algorithm can be used. This algorithm can be written has follow:

$$
w \triangleq w_{t}(S, \dot{S})= \begin{cases}-\lambda_{M} \operatorname{sgn}(S) & \text { if } S \dot{S}>0, \\ -\lambda_{m} \operatorname{sgn}(S) & \text { if } S \dot{S} \geq 0 .\end{cases}
$$

From (Levant 1993) one can find the sufficient conditions which show the finite time convergence onto the real second order sliding set:

$$
\begin{aligned}
& \lambda_{m}>4 \frac{K_{M}}{S_{0}}, \\
& \lambda_{m}>\frac{C_{0}}{k_{m}}, \\
& \lambda_{M}>\frac{K_{M} \lambda_{m}}{k_{m}}+2 \frac{C_{0}}{k_{m}},
\end{aligned}
$$

where:

$$
0<k_{m} \leq|\varphi(t, S, \dot{S})| \leq K_{M} \text { and }|\phi(t, S, \dot{S})|<C_{0}
$$

The finite time convergence of this algorithm based on a Lyapunov function can be found in (Orlov 2009).

\section{$2.3 \quad$ Control strategy}

Previously, three different frames to express the model of the PMSM have been presented. It will be shown that the $f-g$ frame is suitable for sensorless control of PMSM.

The control law is proposed for the position tracking of PMSM. The control is designed from the flatness property of the motor. Indeed, in (Sira-Ramírez 2000) it was shown that PMSM were flat systems, where the flat outputs are the direct currents and the position. For this system to be controlled, it is necessary to design a control loop for the direct currents and another for 
the position. For the current loop, the sliding variable is chosen so that the system has relative degree equal to 1, a Super Twisting algorithm can be used. The sliding variable measurement only is needed and this algorithm reduces the chattering phenomena. For the position control loop, the system is of third degree. A judicious choice of the sliding variable lead to a system with relative degree equal to 2 with respect to the sliding variable which enables the use of a Twisting algorithm, for which the position, the velocity and the acceleration are needed.

For a mechanical sensorless control, based on this described control law, the position and the velocity must be estimated but also the acceleration of the motor. Here, the proposed approach is based on the back-EMF estimation. The electrical equations (9a), (9b) are used to estimate the back-EMF using input voltages and measured currents only. From this estimation, the position and the velocity can be reconstructed for the control. For this purpose, two sliding mode observers are designed. These observers are advantageous in the presence of the nonlinearities of the backEMF. The observers convergence is ensured under the assumption that the time derivatives of these terms are bounded. Moreover these observers ensure the finite time convergence of the estimate that facilitates the proof of the closed-loop stability of the whole control-observer. The proposed control law also needs the estimation of the acceleration to be designed. It is estimated from the mechanical equation (9c) using the estimated position and velocity.

Moreover, the PMSM is not observable at zero velocity, as it is proven in (Ezzat et al. 2010). To overcome the unobservability case, in the sequel, a low-speed strategy to bring the motor states in the observable domain is proposed. Thus, the main objective of this work is the robust position trajectory tracking without any mechanical sensors.

The control scheme is shown Fig. 2.

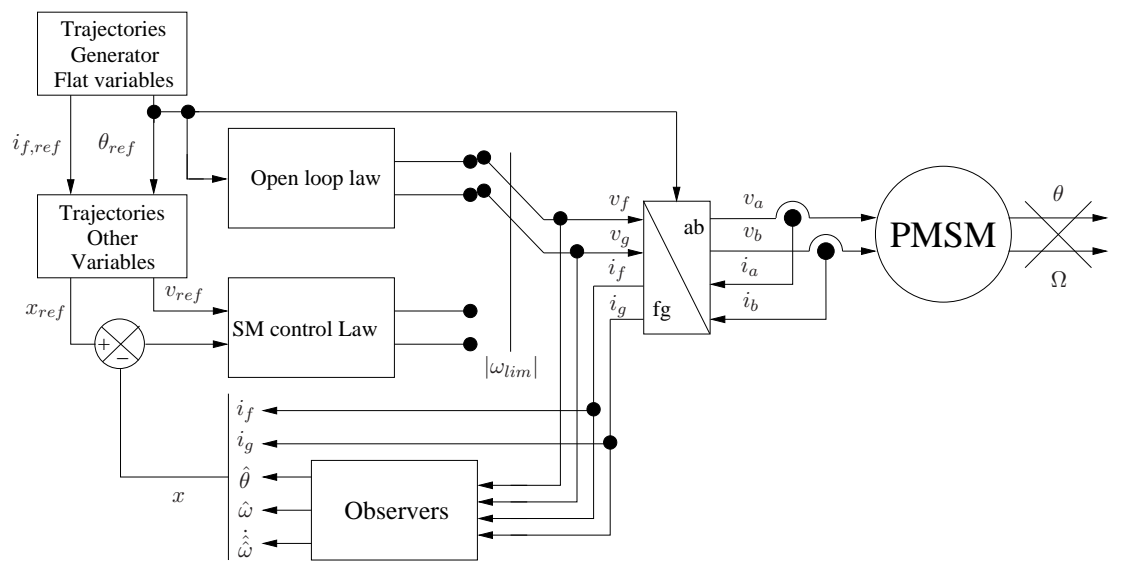

Figure 2. System global scheme.

\section{Mechanical sensorless sliding mode observers}

In this paragraph, sliding mode observers are designed for the estimation of the position, the velocity and the acceleration. The observers are designed in the $(f-g)$ frame. The position and the velocity are estimated using the electrical equations. These estimations are then used for the acceleration estimation based on the mechanical equation.

\subsection{Electrical equations based observers}

\subsubsection{Observer design}

The electrical equations of the motor in the $(f-g)$ frame are given by $(9 \mathrm{a})$ and $(9 \mathrm{~b})$. 
These equations can be represented under the form:

$$
\begin{aligned}
& \dot{x}_{f g}=A_{f g} x_{f g}+u_{f g}+d_{f g}, \\
& y_{f g}=C_{f g} x_{f g}
\end{aligned}
$$

where $x_{f g}=\left[i_{f} i_{g}\right]^{T}, u_{f g}=\left[\frac{1}{L}\left(v_{f}-R i_{f}\right) \frac{1}{L}\left(v_{g}-R i_{g}\right)\right]^{T}$ and,

$$
A_{f g}=\left[\begin{array}{cc}
0 & n_{p} \omega_{r} \\
-n_{p} \omega_{r} & 0
\end{array}\right], d_{f g}=\left[\begin{array}{l}
d_{f} \\
d_{g}
\end{array}\right]=\left[\begin{array}{c}
\frac{K}{L} \omega \sin \left(n_{p} \Delta \theta\right) \\
-\frac{K}{L} \omega \cos \left(n_{p} \Delta \theta\right)
\end{array}\right] \text { and } C_{f g}=\left[\begin{array}{ll}
1 & 0 \\
0 & 1
\end{array}\right]
$$

the disturbance $d_{f g}$ are introduced by the permanent magnets. A second order sliding mode observer is designed. Given that is acts on the sliding variable and its first derivative, the model (23) is rewritten under an augmented form, where the variables $d_{f}$ and $d_{g}$ are the augmented state variables to be estimated:

$$
\begin{aligned}
& \dot{\mathbf{x}}_{f g}=\mathbf{A}_{f g} \mathbf{x}_{f g}+\mathbf{B}_{f g} u_{f g}+\mathbf{d}_{f g}, \\
& \mathbf{y}_{f g}=\mathbf{C}_{f g} \mathbf{x}_{f g}
\end{aligned}
$$

where $\mathbf{x}_{f g}=\left[\begin{array}{llll}i_{f} & i_{g} & d_{f} & d_{g}\end{array}\right]^{T}$ and,

$$
\begin{gathered}
\mathbf{A}_{f g}=\left[\begin{array}{c|c}
A & \mathbf{I}_{2 \times 2} \\
\hline \mathbf{0}_{2 \times 2} & \mathbf{0}_{2 \times 2}
\end{array}\right], \mathbf{B}_{f g}=\left[\frac{\mathbf{I}_{2 \times 2}}{\mathbf{0}_{2 \times 2}}\right], \mathbf{C}_{f g}=\left[\mathbf{I}_{2 \times 2} \mid \mathbf{0}_{2 \times 2}\right] \text { and } \\
\mathbf{d}_{f g}=\left[\begin{array}{c}
\mathbf{0}_{2 \times 1} \\
\frac{K}{L}\left(\dot{\omega} \sin \left(n_{p} \Delta \theta\right)+n_{p} \omega \Delta \omega \cos \left(n_{p} \Delta \theta\right)\right) \\
-\frac{K}{L}\left(\dot{\omega} \cos \left(n_{p} \Delta \theta\right)-n_{p} \omega \Delta \omega \sin \left(n_{p} \Delta \theta\right)\right)
\end{array}\right]
\end{gathered}
$$

where $\Delta \omega=\omega-\omega_{r}$. The sliding mode observer is constructed by replacing the disturbance voltages by the output injection matrix $\chi_{f g}\left(\mathbf{y}_{f g}-\hat{\mathbf{y}}_{f g}\right)$ yet to be defined. A stabilizing linear part $\mathbf{L}_{f g}\left(\mathbf{y}_{f g}-\hat{\mathbf{y}}_{f g}\right)$ is also introduced. The observer is defined by:

$$
\begin{aligned}
& \dot{\hat{\mathbf{x}}}_{f g}=\mathbf{A}_{f g} \hat{\mathbf{x}}_{f g}+\mathbf{B}_{f g} u_{f g}-\chi_{f g}\left(\mathbf{y}_{f g}-\hat{\mathbf{y}}_{f g}\right)-\mathbf{L}_{f g}\left(\mathbf{y}_{f g}-\hat{\mathbf{y}}_{f g}\right), \\
& \hat{\mathbf{y}}_{f g}=\mathbf{C}_{f g} \hat{\mathbf{x}}_{f g} .
\end{aligned}
$$

The output injection matrix $\chi_{f g}\left(\mathbf{y}_{f g}-\hat{\mathbf{y}}_{f g}\right)$ is defined by the Super Twisting algorithm (relative degree equal to 1 w.r.t. the sliding variable):

$$
\chi_{f g}\left(\mathbf{y}_{f g}-\hat{\mathbf{y}}_{f g}\right)=\left[\begin{array}{c}
\lambda_{f}\left|i_{f}-\hat{i}_{f}\right|^{\frac{1}{2}} \operatorname{sgn}\left(i_{f}-\hat{i}_{f}\right) \\
\lambda_{g}\left|i_{g}-\hat{i}_{g}\right|^{\frac{1}{2}} \operatorname{sgn}\left(i_{g}-\hat{i}_{g}\right) \\
\alpha_{f} \operatorname{sgn}\left(i_{f}-\hat{i}_{f}\right) \\
\alpha_{g} \operatorname{sgn}\left(i_{g}-\hat{i}_{g}\right)
\end{array}\right] .
$$

The linear term is defined by:

$$
\mathbf{L}_{f g}=\left[\begin{array}{cc}
l_{f} & 0 \\
0 & l_{g} \\
\hline \mathbf{0}_{2 \times 2}
\end{array}\right]
$$

where $l_{f}$ and $l_{g}$ are adjustable gains yet to be defined. 
The observation errors are defined by the vector:

$$
\boldsymbol{\epsilon}_{f g}=\mathbf{x}_{f g}-\hat{\mathbf{x}}_{f g}=\left[\begin{array}{c}
\epsilon_{f} \\
\epsilon_{g} \\
\epsilon_{d_{f}} \\
\epsilon_{d_{g}}
\end{array}\right]=\left[\begin{array}{c}
i_{f}-\hat{i}_{f} \\
i_{g}-\hat{i}_{g} \\
d_{f}-\hat{d}_{f} \\
d_{g}-\hat{d}_{g}
\end{array}\right]
$$

From equations (23) and (25), the errors dynamics are given by:

$$
\dot{\boldsymbol{\epsilon}}_{f g}=\dot{\mathbf{x}}_{f g}-\dot{\hat{\mathbf{x}}}_{f g}=\left(\mathbf{A}_{f g}-\mathbf{L}_{f g} \mathbf{C}_{f g}\right) \boldsymbol{\epsilon}_{f g}+\mathbf{d}_{f g}-\chi\left(\boldsymbol{\epsilon}_{f g}\right)
$$

Under physical assumption, one can consider that the velocity and the acceleration of the motor are bounded. Thus, the disturbances $d_{f}$ and $d_{g}$ are upperbounded by $\Pi_{d}=$ $\frac{K}{L}\left(|\dot{\omega}|_{\max }+n_{p}|\omega \Delta \omega|_{\max }\right)$. The finite time convergence conditions of the Super Twisting algorithms are satisfied choosing the parameters $\alpha, \lambda$ and the gain matrix $\mathbf{L}_{f g}$ such that they satisfy the conditions given by the Lyapunov function introduced in (18).

After a finite time, a second order sliding motion occurs on the sliding manifold $\left\{\epsilon_{f g}=\dot{\epsilon}_{f g}=0\right\}$ implying:

$$
\left\{\begin{array}{l}
\dot{\epsilon}_{f}=0, \\
\dot{\epsilon}_{g}=0, \\
\dot{\epsilon}_{d_{f}}=0=\frac{K}{L}\left(\dot{\omega} \sin \left(n_{p} \Delta \theta\right)+n_{p} \omega \Delta \omega \cos \left(n_{p} \Delta \theta\right)\right)-\alpha_{f} \operatorname{sgn}\left(\epsilon_{f}\right), \\
\dot{\epsilon}_{d_{g}}=0=-\frac{K}{L}\left(\dot{\omega} \cos \left(n_{p} \Delta \theta\right)-n_{p} \omega \Delta \omega \sin \left(n_{p} \Delta \theta\right)\right)-\alpha_{g} \operatorname{sgn}\left(\epsilon_{g}\right),
\end{array}\right.
$$

whether:

$$
\left\{\begin{aligned}
\hat{d}_{f} & =\frac{K}{L} \omega \sin \left(n_{p} \Delta \theta\right), \\
\hat{d}_{g} & =-\frac{K}{L} \omega \cos \left(n_{p} \Delta \theta\right), \\
\alpha_{f} \operatorname{sgn}_{e q}\left(\epsilon_{f}\right) & =\frac{\dot{\omega}}{\omega} \hat{d}_{f}-n_{p} \hat{d}_{g} \Delta \omega, \\
\alpha_{g} \operatorname{sgn}_{e q}\left(\epsilon_{g}\right) & =\frac{\dot{\omega}}{\omega} \hat{d}_{g}+n_{p} \hat{d}_{f} \Delta \omega,
\end{aligned}\right.
$$

where $\alpha_{*} \operatorname{sgn}_{e q}\left(\epsilon_{*}\right)$ with $* \in\{f, g\}$ represents the equivalent output injections obtained after filtering.

\subsubsection{Position and velocity reconstruction}

\section{Position estimation:}


From equations (28a) and (28b), the estimation $\Delta \theta_{\text {est }}$ of $\Delta \theta$ is given by:

$$
\Delta \theta_{\text {est }}=\left\{\begin{array}{cc}
\text { undefined } & \text { if } \quad \omega=0, \\
\frac{1}{n_{p}} \arctan \left(\frac{\hat{d}_{f}}{\hat{d}_{g}}\right) & \text { if } \quad \hat{c}>0, \\
\pi / 2 n_{p} & \text { if } \quad \hat{s}=1, \\
-\pi / 2 n_{p} & \text { if } \hat{s}=-1, \\
\frac{1}{n_{p}}\left(\arctan \left(\frac{\hat{d}_{f}}{\hat{d}_{g}}\right)-\pi\right) & \text { if } \hat{c}<0, \\
\frac{1}{n_{p}}\left(\arctan \left(\frac{\hat{d}_{f}}{\hat{d}_{g}}\right)+\pi\right) & \text { and } \hat{s}>0, \\
& \text { if } \hat{c}<0, \\
\text { and } \hat{s}<0,
\end{array}\right.
$$

where $\hat{c}=\hat{d}_{g} / \sqrt{\hat{d}_{f}^{2}+\hat{d}_{g}^{2}}, \hat{s}=\hat{d}_{f} / \sqrt{\hat{d}_{f}^{2}+\hat{d}_{g}^{2}}$ are the estimations of $\cos \left(n_{p} \Delta \theta\right)$ and $\sin \left(n_{p} \Delta \theta\right)$, respectively. The output value of the function arctan computed using (29) takes values in the interval $]-\frac{\pi}{n_{p}}, \frac{\pi}{n_{p}}[$, leading to discontinuous output.

Velocity estimation: From equations (28a) and (28b) the estimation of the velocity module could be reconstructed as:

$$
\left|\omega_{e s t}\right|=\sqrt{\hat{d}_{f}^{2}+\hat{d}_{g}^{2}}
$$

This expression gives only the absolute value of the velocity.

However, substituting $\omega$ and $\dot{\omega}$ in equations (28c) and (28d), one has:

$$
\Delta \omega_{e s t}=\frac{1}{n_{p}} \frac{\alpha_{g} \operatorname{sgn}_{e q}\left(\epsilon_{g}\right) \hat{d}_{f}-\alpha_{f} \operatorname{sgn}_{e q}\left(\epsilon_{f}\right) \hat{d}_{g}}{\hat{d}_{f}^{2}+\hat{d}_{g}^{2}} .
$$

From equations (30) and (31), one deduces the velocity estimation:

$$
\omega_{e s t}=\omega_{r}+\Delta \omega_{e s t}
$$

Remark 3.1 Note that the acceleration could also be obtained from equations (28c) and (28d). The discontinuities of the Super Twisting algorithm acting on these equations make them more sensitive to chattering phenomenon, the resulting expression for the acceleration is ill conditioned. An alternative method is discussed in the next section.

\subsection{Mechanical equation based observer}

In order to apply a second order the sliding mode control, one proposes to design an observer to estimate the acceleration. Therefore the mechanical equation in the $(f-g)$ frame is rewritten replacing the position and the velocity by their estimates

$$
\dot{\omega}=\frac{1}{J}\left(K\left(i_{f} \sin \left(n_{p} \Delta \theta_{e s t}\right)+i_{g} \cos \left(n_{p} \Delta \theta_{e s t}\right)-f_{v} \omega_{e s t}-C_{r}\right) .\right.
$$

The load torque is considered as a perturbation denoted:

$$
d_{\omega_{e s t}}=-\frac{C_{r}}{J}
$$


The augmented system is defined by:

$$
\begin{aligned}
& \dot{\mathbf{x}}_{\omega_{e s t}}=\mathbf{A}_{\omega_{e s t}} \mathbf{x}_{\omega_{e s t}}+\boldsymbol{B}_{\omega_{e s t}} u_{\omega_{e s t}}+\boldsymbol{d}_{\omega_{e s t}}, \\
& \mathbf{y}_{\omega_{e s t}}=\mathbf{C}_{\omega_{e s t}} \mathbf{x}_{\omega_{e s t}},
\end{aligned}
$$

where $\mathbf{x}_{\omega_{e s t}}=\left[\omega_{\text {est }} d_{\omega_{e s t}}\right]^{T}, u_{\omega_{e s t}}=\frac{K}{J}\left(i_{f} \sin \left(n_{p} \Delta \theta_{e s t}\right)+i_{g} \cos \left(n_{p} \Delta \theta_{e s t}\right)\right)$ and,

$$
\boldsymbol{A}_{\omega_{e s t}}=\left[\begin{array}{cc}
-\frac{f_{v}}{J} & 1 \\
0 & 0
\end{array}\right], \boldsymbol{B}_{\omega_{e s t}}=\left[\begin{array}{l}
1 \\
0
\end{array}\right], \boldsymbol{d}_{\omega_{e s t}}=\left[\begin{array}{c}
0 \\
-\frac{1}{J} \frac{d C_{r}}{d t}
\end{array}\right] \text { and } \boldsymbol{C}_{\omega_{e s t}}=\left[\begin{array}{ll}
1 & 0
\end{array}\right]
$$

The observer under the augmented form becomes:

$$
\begin{aligned}
& \dot{\hat{\mathbf{x}}}_{\omega_{e s t}}=\mathbf{A}_{\omega_{\text {est }}} \hat{\mathbf{x}}_{\omega_{e s t}}+\mathbf{B}_{\omega_{e s t}} u_{\omega_{e s t}}+\chi_{\omega_{e s t}}\left(\mathbf{y}_{\omega_{e s t}}-\hat{\mathbf{y}}_{\omega_{e s t}}\right)+\mathbf{l}_{\omega_{e s t}}\left(\mathbf{y}_{\omega_{e s t}}-\hat{\mathbf{y}}_{\omega_{e s t}}\right), \\
& \hat{\mathbf{y}}_{\omega_{e s t}}=\mathbf{C}_{\omega_{e s t}} \hat{\mathbf{x}}_{\omega_{e s t}},
\end{aligned}
$$

where

$$
\begin{gathered}
\chi_{\omega_{\text {est }}}\left(\mathbf{y}_{\omega_{\text {est }}}-\hat{\mathbf{y}}_{\omega_{\text {est }}}\right)=\left[\begin{array}{c}
\lambda_{\omega_{\text {est }}} \mid \mathbf{y}_{\omega_{\text {est }}}-\hat{\mathbf{y}}_{\omega_{\text {est }}}{ }^{\frac{1}{2}} \operatorname{sgn}\left(\mathbf{y}_{\omega_{\text {est }}}-\hat{\mathbf{y}}_{\omega_{\text {est }}}\right) \\
\alpha_{\omega_{\text {est }}} \operatorname{sgn}\left(\mathbf{y}_{\omega_{\text {est }}}-\hat{\mathbf{y}}_{\omega_{\text {est }}}\right)
\end{array}\right], \\
\mathbf{l}_{\omega_{\text {est }}}\left(\mathbf{y}_{\omega_{\text {est }}}-\hat{\mathbf{y}}_{\omega_{\text {est }}}\right)=\left[\begin{array}{c}
l_{\omega} \\
0
\end{array}\right]\left(\mathbf{y}_{\omega_{\text {est }}}-\hat{\mathbf{y}}_{\omega_{\text {est }}}\right) .
\end{gathered}
$$

The observation error is defined by:

$$
\epsilon_{\omega_{e s t}}=\mathbf{x}_{\omega_{e s t}}-\hat{\mathbf{x}}_{\omega_{e s t}}=\left[\begin{array}{c}
\epsilon_{\mathbf{y}_{\omega_{e s t}}} \\
\epsilon_{d_{\omega_{e s t}}}
\end{array}\right]=\left[\begin{array}{c}
\mathbf{y}_{\omega_{e s t}}-\hat{\mathbf{y}}_{\omega_{e s t}} \\
d_{\omega_{e s t}}-\hat{d}_{\omega_{e s t}}
\end{array}\right]
$$

leading to the dynamics:

$$
\dot{\epsilon}_{\omega_{e s t}}=\dot{\mathbf{x}}_{\omega_{e s t}}-\dot{\hat{\mathbf{x}}}_{\omega_{e s t}}=\left(\mathbf{A}_{\omega_{e s t}}-\mathbf{l}_{\omega_{e s t}} \mathbf{C}_{\omega_{e s t}}\right) \epsilon_{\omega_{e s t}}+\mathbf{d}_{\omega_{e s t}}-\chi_{\omega_{e s t}}\left(\epsilon_{\mathbf{y}_{\omega_{e s t}}}\right) \text {. }
$$

Under the assumption that the coefficient of Coulomb friction is differentiable, and that the derivative of $C_{r}$ is bounded (i.e. $\left|\frac{d C_{r}}{d t}\right|_{\max }<\Pi_{C_{r}}$ where $\Pi_{C_{r}}$ is a positive constant), the finite time convergence of the observer is guaranteed choosing the gains $\alpha_{\omega_{e s t}}, \lambda_{\omega_{e s t}}$, and $l_{\omega_{\text {est }}}$ satisfying the conditions of the Lyapunov function (20). Thus after finite time, one has $\dot{\epsilon}_{\omega_{e s t}}=\epsilon_{\omega_{e s t}}=0$, leading to the estimation of the acceleration $\dot{\omega}_{\text {est }}$. Note that at the same time the load torque is also estimated.

This section has shown a method for the estimation of the position, the velocity and the acceleration. The next section is devoted to the control law design.

\section{Sliding mode control for the PMSM}

The control law is designed considering that the PMSM model is flat. The flatness theory was introduced in (Fliess et al. 1995). A system is said flat if the states and the inputs of the system can be expressed from the flat outputs and a finite number of their time derivatives only. The flatness of the PMSM was shown in (Sira-Ramírez 2000) in the rotating $(d-q)$ frame, without Coulomb friction (i.e. unperturbed), with the flat outputs $\theta$ and $i_{d}$. To be transformed into the $(f-g)$ frame, it suffices to apply the transformation (4) using $U(\Delta \theta)$ instead of $U(\theta)$, leading to the flat outputs in the $(f-g)$ frame $Y_{f g}=\left(y_{f g, 1}, y_{f g, 2}\right)=\left(\theta, i_{f} \cos \left(n_{p} \Delta \theta\right)+i_{g} \sin \left(n_{p} \Delta \theta\right)\right)$

Flatness property allows defining easily a reference trajectory (denoted $\Gamma_{r}$ ), satisfying the system dynamics. Considering that $\theta_{r}$ is the reference position to be tracked, the reference 
model of the motor in the $(f-g)$ frame is obtained, replacing $\theta$ by $\theta_{r}$ in the model and is given by:

$$
\left\{\begin{aligned}
L \frac{d i_{f, r}}{d t} & =v_{f, r}-R i_{f, r}+n_{p} L \omega_{r} i_{g, r} \\
L \frac{d i_{g, r}}{d t} & =v_{g, r}-R i_{g, r}-n_{p} L \omega_{r} i_{f, r}-K \omega_{r} \\
J \frac{d \omega_{r}}{d t} & =K i_{g, r}-f_{v} \omega_{r} \\
\frac{d \theta_{r}}{d t} & =\omega_{r} .
\end{aligned}\right.
$$

The flat outputs reference trajectories are defined by $\theta_{r}$ and $i_{f, r}$. From these outputs, all the reference variables can be defined using the equations:

$$
\begin{aligned}
& \omega_{r}=\frac{d \theta_{r}}{d t} \\
& i_{g, r}=\frac{1}{K}\left(J \frac{d^{2} \theta_{r}}{d t^{2}}+f_{v} \frac{d \theta_{r}}{d t}\right), \\
& v_{f, r}=L \frac{d i_{f, r}}{d t}+R i_{f, r}-\frac{N L}{K} \frac{d \theta_{r}}{d t}\left(J \frac{d^{2} \theta_{r}}{d t^{2}}+f_{v} \frac{d \theta_{r}}{d t}\right), \\
& v_{g, r}=\frac{J L}{K} \frac{d^{3} \theta_{r}}{d t^{3}}+\frac{1}{K}\left(L f_{v}+R J\right) \frac{d^{2} \theta_{r}}{d t^{2}}+\left(\frac{R f_{v}}{K}+K+N L i_{f, r}\right) \frac{d \theta_{r}}{d t} .
\end{aligned}
$$

The dynamics of the tracking error:

$$
e_{f g}=\left[e_{f}, e_{g}, e_{\omega}, e_{\theta}\right]^{T}=\left[\begin{array}{c}
i_{f} \cos \left(n_{p} e_{\theta}\right)+i_{g} \sin \left(n_{p} e_{\theta}\right)-i_{f r} \\
-i_{f} \sin \left(n_{p} e_{\theta}\right)+i_{g} \cos \left(n_{p} e_{\theta}\right)-i_{g r} \\
\Delta \omega \\
\Delta \theta
\end{array}\right]
$$

are given by:

$$
\left\{\begin{array}{l}
\dot{e}_{f}=\frac{1}{L}\left(\bar{v}_{f}-R e_{f}+n_{p} L\left(e_{\omega} e_{g}+e_{\omega} i_{g, r}+e_{g} \omega_{r}\right)\right) \\
\dot{e}_{g}=\frac{1}{L}\left(\bar{v}_{g}-R e_{g}+n_{p} L\left(e_{\omega} e_{f}+e_{\omega} i_{f, r}+e_{f} \omega_{r}\right)-K e_{\omega}\right) \\
\dot{e}_{\omega}=\frac{1}{J}\left(K e_{g}-f_{v} e_{\omega}-C_{r}\right) \\
\dot{e}_{\theta}=e_{\omega}
\end{array}\right.
$$

with:

$$
\left[\begin{array}{c}
\bar{v}_{f} \\
\bar{v}_{g}
\end{array}\right]^{T}=U\left(e_{\theta}\right)\left[\begin{array}{l}
v_{f} \\
v_{g}
\end{array}\right]^{T}-\left[\begin{array}{l}
v_{f, r} \\
v_{g, r}
\end{array}\right]^{T} .
$$

The flat variables can be expressed as a function of the inputs:

$$
\left\{\begin{aligned}
\dot{e}_{f} & =\frac{1}{L} \bar{v}_{f}+\mu_{1}\left(e_{f g}\right) \\
e_{\theta}^{(3)} & =\frac{K}{J L} \bar{v}_{g}+\mu_{2}\left(e_{f g}\right)+\frac{f_{v}}{J^{2}} C_{r}-\frac{1}{J} \frac{d C_{r}}{d t}
\end{aligned}\right.
$$

where:

$$
\begin{aligned}
& \mu_{1}\left(e_{f g}\right)=\frac{1}{L}\left(-R e_{f}+N L\left(e_{\omega} e_{g}+e_{\omega} i_{g, r}+e_{g} \omega_{r}\right)\right) \\
& \mu_{2}\left(e_{f g}\right)=-\frac{K}{J L}\left(R e_{g}+N L\left(e_{\omega} e_{f}+e_{\omega} i_{f, r}+e_{f} \omega_{r}\right)+K e_{\omega}\right)-\frac{f_{v}}{J^{2}}\left(K e_{f}-f_{v} e_{\omega}\right) .
\end{aligned}
$$




\subsection{Observation based state feedback control}

The control law is designed using the state estimation obtained Section 3. The error vector is defined between the estimated variables and the reference trajectories by:

$$
\xi=\left[\xi_{f}, \xi_{g}, \xi_{\omega}, \xi_{\theta}\right]^{T},
$$

with

$$
\left[\begin{array}{c}
\xi_{f} \\
\xi_{g} \\
\xi_{\omega} \\
\xi_{\theta}
\end{array}\right]=\left[\begin{array}{c}
i_{f} \cos \left(N\left(\theta_{\text {est }}-\theta_{r}\right)\right)+i_{g} \sin \left(N\left(\theta_{\text {est }}-\theta_{r}\right)\right)-i_{f, r} \\
-i_{f} \sin \left(N\left(\theta_{\text {est }}-\theta_{r}\right)\right)+i_{g} \cos \left(N\left(\theta_{e s t}-\theta_{r}\right)\right)-i_{g, r} \\
\omega_{e s t}-\omega_{r} \\
\theta_{e s t}-\theta_{r}
\end{array}\right]
$$

Leading to:

$$
\left\{\begin{aligned}
\dot{\xi}_{f} & =\frac{1}{L} \bar{v}_{f}+\mu_{1}\left(\xi_{f g}\right), \\
\xi_{\theta}^{(3)} & =\frac{K}{J L} \bar{v}_{g}+\mu_{2}\left(\xi_{f g}\right)+\frac{f_{v}}{J^{2}} C_{r}-\frac{1}{J} \frac{d C_{r}}{d t},
\end{aligned}\right.
$$

where:

$$
\begin{aligned}
& \mu_{1}\left(\xi_{f g}\right)=\frac{1}{L}\left(-R \xi_{f}+N L\left(\xi_{\omega} \xi_{g}+\xi_{\omega} i_{g, r}+\xi_{g} \omega_{r}\right)\right) \\
& \mu_{2}\left(\xi_{f g}\right)=-\frac{K}{J L}\left(R \xi_{g}+N L\left(e_{\omega} \xi_{f}+\xi_{\omega} i_{f, r}+\xi_{f} \omega_{r}\right)+K \xi_{\omega}\right)-\frac{f_{v}}{J^{2}}\left(K \xi_{f}-f_{v} \xi_{\omega}\right) .
\end{aligned}
$$

It is desired to ensure the convergence to the origin of the system (45). In this section, the perturbations are unknown, but supposed bounded as well as their time derivatives. It is then important to propose a control law which guarantee the convergence despite the perturbations. From equation (45), one has to design a control law for the $i_{f}$ current tracking and a law for the position tracking.

\subsection{1 $i_{f}$ current tracking}

In order to ensure the current tracking, the following sliding variable is chosen:

$$
S_{f}=\xi_{f}
$$

From equation (45a), the time derivative of this variable is given by:

$$
\dot{S}_{f}=\dot{\xi}_{f}=\frac{1}{L} \bar{v}_{f}+\mu_{1}\left(\xi_{f g}\right) .
$$

Since the control appears in the first derivative, the system has a relative degree equal to 1 w.r.t. the sliding variable. A first order sliding mode algorithm would have been sufficient in this case. However the chattering phenomena is more important if the discontinuous control is applied directly on the time derivative of the current. It is proposed here to use a second order algorithm so that the discontinuous action is applied to the second derivative. The Super Twisting algorithm which require the knowledge of $\xi_{f}$, only, is used.

Define first a static state feedback $\bar{v}_{f}$ :

$$
\frac{1}{L} \bar{v}_{f}=-\mu_{1}\left(\xi_{f} g\right)+u_{s t}\left(S_{f}\right),
$$


where $u_{s t}\left(S_{f}\right)$ is the Super Twisting algorithm defined in (13). The gains $\alpha$ and $\lambda$ are determined considering the system (16) without perturbations.

The time derivative of the Lyapunov function (20) is given by:

$$
\dot{V}=|\psi|^{-1} \psi^{T}\left(M^{T} P+P M\right) \psi
$$

with $P$ a symmetric positive definite and $M=\left[\begin{array}{ll}-\lambda & 1 \\ -\alpha & 0\end{array}\right]$.

The gains $\alpha$ and $\lambda$ must be chosen such that:

$$
-Q_{1}=M^{T} P+P M
$$

with $Q_{1}$ positive definite.

This condition is satisfied if the matrix $M$ is Hurwitz, whether:

$$
\begin{aligned}
& \operatorname{tr}(M)=-\lambda, \\
& \operatorname{det}(M)=\alpha .
\end{aligned}
$$

Choosing $\alpha$ and $\lambda$ strictly positive, this condition is satisfied.

\subsubsection{Position tracking}

In order to obtain a system with relative degree equal to 2 , the sliding variable is defined by:

$$
S_{\theta}=k \xi_{\theta}+\dot{\xi}_{\theta}
$$

with $k>0$. Moreover, the sliding variable depends on the position and the velocity only, and do not depends on the motor parameters. The successive time derivatives of $S_{\theta}$ are:

$$
\begin{aligned}
\dot{S}_{\theta} & =k \dot{\xi}_{\theta}+\ddot{\xi}_{\theta}, \\
\ddot{S}_{\theta} & =k \dot{\xi}_{\omega}+\ddot{\xi}_{\omega} \\
& =\frac{k}{J}\left(K \xi_{g}-f_{v} e_{\omega}\right)+\frac{K}{J L} \bar{v}_{g}+\mu_{2}\left(\xi_{f g}\right)-\left(\frac{k}{J}-\frac{f_{v}}{J^{2}}\right) C_{r}-\frac{1}{J} \frac{d C_{r}}{d t} .
\end{aligned}
$$

In (Nollet et al. 2008), the Sampled Twisting algorithm was used. This algorithm does not require the time derivative of the sliding variable, but it uses the difference over a sampling period of the variable, which is very sensitive to measurement noise. Thus in the section 2.2 , we have introduced the Twisting algorithm to treat systems of relative degree equal to 2.

Choosing the control input $\bar{v}_{q}$ as follow:

$$
\frac{K}{J L} \bar{v}_{g}=-\frac{k}{J}\left(K \xi_{g}-f_{v} \xi_{\omega}\right)-\mu_{2}\left(\xi_{f g}\right)+w_{t}\left(S_{\theta}, \dot{S}_{\theta}\right),
$$

where $w_{t}\left(S_{\theta}, \dot{S}_{\theta}\right)$ is the twisting algorithm defined by equation (21).

The second time derivative of the sliding variable (52) can be written as:

$$
\ddot{S}_{\theta}=\phi_{1}(t)+w_{t}\left(S_{\theta}, \dot{S}_{\theta}\right)
$$

where:

$$
\phi_{1}(t)=-\left(\frac{k}{J}-\frac{f_{v}}{J^{2}}\right) C_{r}-\frac{1}{J} \frac{d C_{r}}{d t} .
$$


Equation (55) is expressed with the form given in equation (11). Thus the finite time convergence towards the surface $S_{\theta}=0$ is guaranteed if the conditions (22) for the controller gains $\lambda_{m}$ and $\lambda_{M}$ :

$$
\begin{aligned}
& \lambda_{m}>\left|\left(\frac{k}{J}-\frac{f_{v}}{J^{2}}\right) C_{r}-\frac{1}{J} \frac{d C_{r}}{d t}\right|_{\text {max }}, \\
& \lambda_{M}>\lambda_{m}+2\left|\left(\frac{k}{J}-\frac{f_{v}}{J^{2}}\right) C_{r}-\frac{1}{J} \frac{d C_{r}}{d t}\right|_{\max },
\end{aligned}
$$

holds.

Therefore, the presented control law guarantees exponential convergence to 0 of the tracking error, in the case where the estimation from the observers are equal to the real variables. Note that this control law is robust with respect to disturbances and parameter uncertainties. However the system is nonlinear. The separation principle is then not valid. In the next subsection we will study the convergence of the control law and the observers together.

\subsection{Closed loop stability}

The state dynamics of the complete system including the tracking errors as well as the estimation errors

$$
\Xi \triangleq\left[e_{f}, e_{g}, e_{\omega}, e_{\theta}, \epsilon_{f}, \epsilon_{g}, \epsilon_{d_{f}}, \epsilon_{d_{g}} \epsilon_{\mathbf{y}_{\omega_{e s t}}}, \epsilon_{d_{\omega_{e s t}}}\right]^{T},
$$

are given by:

$$
\dot{\Xi}=\left[\begin{array}{c}
-\frac{R}{L} e_{f}+n_{p}\left(e_{\omega} e_{g}+e_{\omega} i_{g, r}+e_{g} \omega_{r}\right) \\
-\frac{R}{L} e_{g}+n_{p}\left(e_{\omega} e_{f}+e_{\omega} i_{f, r}+e_{f} \omega_{r}\right)-\frac{K}{L} e_{\omega} \\
\frac{1}{J}\left(K e_{g}-f_{v} e_{\omega}-C_{r}\right) \\
e_{3} \\
-l_{f} \epsilon_{f}+n_{p} \omega_{r} \epsilon_{g} \\
-n_{p} \omega_{r} \epsilon_{f}-l_{g} \epsilon_{g} \\
\frac{K}{L}\left(\dot{\omega} \sin \left(n_{p} e_{\theta}\right)+n_{p}\left(e_{\omega}+\omega_{r}\right) e_{\omega} \cos \left(n_{p} e_{\theta}\right)\right) \\
-\frac{K}{L}\left(\dot{\omega} \cos \left(n_{p} e_{\theta}\right)-n_{p}\left(e_{\omega}+\omega_{r}\right) e_{\omega} \sin \left(n_{p} e_{\theta}\right)\right) \\
\left(-\frac{f_{v}}{J}-l_{\omega_{e s t}}\right) \epsilon_{\mathbf{y}_{\omega_{e s t}}}+\epsilon_{d_{g}} \\
-\frac{1}{J} \frac{d C_{r}}{d t}
\end{array}\right]+\left[\begin{array}{c}
\frac{1}{L} \bar{v}_{f} \\
\frac{1}{L} \bar{v}_{g} \\
0 \\
0 \\
w_{s t, 1}\left(\epsilon_{f}\right) \\
w_{s t, 1}\left(\epsilon_{g}\right) \\
\dot{w}_{s t, 2}\left(\epsilon_{f}\right) \\
\dot{w}_{s t, 2}\left(\epsilon_{g}\right) \\
w_{s t, 1}\left(\epsilon_{\mathbf{y}_{\omega s t}}\right) \\
\dot{w}_{s t, 2}\left(\epsilon_{\mathbf{y}_{\omega_{e s t}}}\right)
\end{array}\right],
$$

where:

$$
\dot{\omega}=\frac{1}{J}\left(K\left(i_{f} \sin \left(-N e_{\theta}\right)+i_{g} \cos \left(-N e_{\theta}\right)\right)-f_{v}\left(e_{\omega}+\omega_{r}\right)-C_{r}\right) .
$$

To prove the exponential convergence of the system (59), one has to show that the trajectories of the complete system (59) remain bounded on a finite time interval. Indeed, if the system is bounded, the trajectories are maintained in a compact until the observers converge. Then since the designed observers converge in finite time, after this finite time the system will behave exactly like the system controlled with the state feedback described previously. To prove that the system is bounded the estimated variables $\xi$ have to be written as a function of the system states $\Xi$, the reference trajectory $\Gamma_{r}$ and the control inputs.

$$
\left[\begin{array}{l}
\xi_{f} \\
\xi_{g}
\end{array}\right]=U\left(\Delta \theta_{e s t}\right)\left[\begin{array}{l}
\hat{i}_{f} \\
\hat{i}_{g}
\end{array}\right]-\left[\begin{array}{l}
i_{f, r} \\
i_{g, r}
\end{array}\right]
$$


with:

$$
\left\{\begin{aligned}
\Delta \theta_{e s t} & =-\frac{1}{n_{p}} \arctan \left(\frac{\hat{d}_{f}}{\hat{d}_{g}}\right) \\
\hat{i}_{f} & =\epsilon_{f}+i_{f}=\epsilon_{f}+e_{f}+i_{f, r} \\
\hat{i}_{g} & =\epsilon_{g}+i_{g}=\epsilon_{g}+e_{g}+i_{g, r}
\end{aligned}\right.
$$

Similarly:

$$
\left[\begin{array}{l}
\xi_{\omega} \\
\xi_{\theta}
\end{array}\right]=\left[\begin{array}{c}
\omega_{\text {est }} \\
\theta_{\text {est }}
\end{array}\right]-\left[\begin{array}{c}
\omega_{r} \\
\theta_{r}
\end{array}\right]=\left[\begin{array}{c} 
\pm \frac{L}{K} \sqrt{\hat{d}_{f}^{2}+\hat{d}_{g}^{2}} \\
-\frac{1}{N} \arctan \left(\frac{\hat{d}_{f}}{\hat{d}_{g}}\right)
\end{array}\right]-\left[\begin{array}{c}
\omega_{r} \\
\theta_{r}
\end{array}\right]
$$

After substitution in the system (59), of the control equations $\bar{v}_{f}$ and $\bar{v}_{g}$ with the expressions (60) and (62) of the errors between the estimated variables and the reference variables, one obtain a model depending on the states, the desired trajectories and the control inputs:

$$
\dot{\Xi}=F\left(\Xi, \Gamma_{r}, w_{s t, 1}\left(\epsilon_{f}\right), w_{s t, 1}\left(\epsilon_{g}\right), \dot{w}_{s t, 2}\left(\epsilon_{f}\right), \dot{w}_{s t, 2}\left(\epsilon_{g}\right), w_{s t, 1}\left(\epsilon_{\mathbf{y}_{\omega_{e s t}}}\right), \dot{w}_{s t, 2}\left(\epsilon_{\mathbf{y}_{\omega_{e s t}}}\right)\right) .
$$

Without loss of generalities, one can assume that the chosen reference trajectory is bounded. One has then, using (60):

$$
\left\{\begin{array}{l}
\xi_{f}=O\left(\epsilon_{f}+e_{f}\right) \\
\xi_{g}=O\left(\epsilon_{g}+e_{g}\right)
\end{array}\right.
$$

Since the current $i_{f}$ and $i_{g}$ are saturated, as well as their estimates values $\hat{i}_{f}$ and $\hat{i}_{g}$, the tracking errors of the currents $e_{f}$ and $e_{g}$ and estimation errors $\epsilon_{f}$ and $\epsilon_{g}$ are bounded. Thus, the error variables $\xi_{f}$ and $\xi_{g}$ are bounded. Moreover, the Super Twisting and the Twisting algorithms are bounded.

The system can be written as $\dot{\Xi}=f(\Xi)+g$. Thereby, including all the dominations in the complete system, one obtain the inequalities:

$$
\|\dot{\Xi}\| \leq Q\|\Xi\|+\bar{g}
$$

where $Q$ and $g$ are positive constants.

Integrating (65) one obtain:

$$
\|\Xi(t)\| \leq\|\Xi(0)\|+\int_{0}^{t}(Q\|\Xi(\tau)\|+\bar{g}) d \tau
$$

Applying the Gronwall lemma, one has:

$$
\|\Xi(t)\| \leq\|\Xi(0)\| \exp (C t)+\frac{\bar{g}}{Q} \exp (C t-1)
$$

where $C$ is a positive constant.

Therefore, the complete state system $\Xi$ is bounded. This proves the exponential convergence of the mechanical sensorless control law. 


\section{Experimental results}

\subsection{Open-loop strategy}

As mentioned Section 3, the position is not identifiable at standstill. To overcome the problem, a low speed scenario is described. If the motor velocity is lower than a certain velocity $\left|\omega_{\text {lim }}\right|$, the motor is driven in open loop. The following control law in the rotating reference frame is used:

$$
v= \begin{cases}\sqrt{R^{2}+\left(N \omega_{\text {ref }} L\right)^{2} I_{\max }^{2}+\left(K \omega_{\text {ref }}\right)^{2}}, & \text { if } v<V_{\max } \\ V_{\text {max }}, & \text { else }\end{cases}
$$

$I_{\max }$ is the current limit, $V_{\max }$ is the voltage limit. $v$ is applied to the motor using the transformation $v_{a}=v \cos \left(N \theta_{\text {ref }}\right)$ and $v_{b}=v \sin \left(N \theta_{\text {ref }}\right)$. Because this method does not rely on position and velocity sensors or on estimates of these variables, we will refer to the control algorithm as the open-loop controller.

When the PMSM is controlled in open-loop, it can be assumed that $\Omega_{r} \simeq \Omega$ as long as the motor keeps synchronism. This condition can be verified without a sensor because loss of synchronism results in a stalled motor and/or considerable vibrations.

The open-loop controller is useful at low speed, but falls short of what would be desired from a sensorless control law. In particular, this strategy is not able to achieve great acceleration profiles and the motor cannot operate in certain resonant region.

\section{$5.2 \quad$ Reference trajectory}

In order to shown the performances of the proposed algorithm, a reference trajectory has been designed. Thanks to the flatness properties, the reference trajectory is completely determined from the flat outputs. It is then sufficient to determine $\theta_{r}$ and $i_{f, r}$. Form these variables, all the remaining reference variables are computable.

The objective is to bring the motor position from a position $\theta_{r}\left(t_{i}\right)=\theta_{i}$ to a position $\theta_{r}\left(t_{f}\right)=\theta_{f}$ using smooth dynamics without discontinuities. The following constraints are chosen:

$$
\left\{\begin{array}{c}
\theta_{r}\left(t_{i}\right)=\theta_{r i}, \quad \theta_{r}\left(t_{f}\right)=\theta_{r f}, \\
\dot{\theta}_{r}\left(t_{i}\right)=0, \quad \dot{\theta}_{r}\left(t_{f}\right)=0, \\
\ddot{\theta}_{r}\left(t_{i}\right)=0, \quad \ddot{\theta}_{r}\left(t_{f}\right)=0, \\
\theta_{r}^{(3)}\left(t_{i}\right)=0, \quad \dot{\theta}_{r}^{(3)}\left(t_{f}\right)=0 .
\end{array}\right.
$$

Having 8 constraints, the minimal polynomial must be of degree 7 :

$$
\begin{aligned}
\theta_{r}(t)=\theta_{r i}+\left(\theta_{r f}-\theta_{r i}\right)( & a_{0} \Delta(t)^{7}+a_{6} \Delta(t)^{6}+a_{2} \Delta(t)^{5}+a_{3} \Delta(t)^{4} \\
& \left.+a_{4} \Delta(t)^{3}+a_{5} \Delta(t)^{2}+a_{6} \Delta(t)+a_{7}\right),
\end{aligned}
$$

with:

$$
\Delta(t)=\frac{t-t_{i}}{t_{f}-t_{i}}
$$

and:

$$
\begin{gathered}
a_{0}=20, a_{1}=-70, a_{2}=84, a_{3}=-35 \\
a_{4}=0, \quad a_{5}=0, \quad a_{6}=0, \quad a_{7}=0 .
\end{gathered}
$$

The direct reference current is defined to be null, in order to minimize the Joule losses and 
maximize the motor torque (Bodson et al. 1993), it leads to $i_{f, r}=0 A$. The trajectory of the current could also have been chosen as in (Verl and Bodson 1998), but the optimization is not the goal of this article. From these two variables, $\omega_{r}, \dot{\omega}_{r}$ and $i_{g, r}$ are determined from (38).

\subsection{Test-Bench Description}

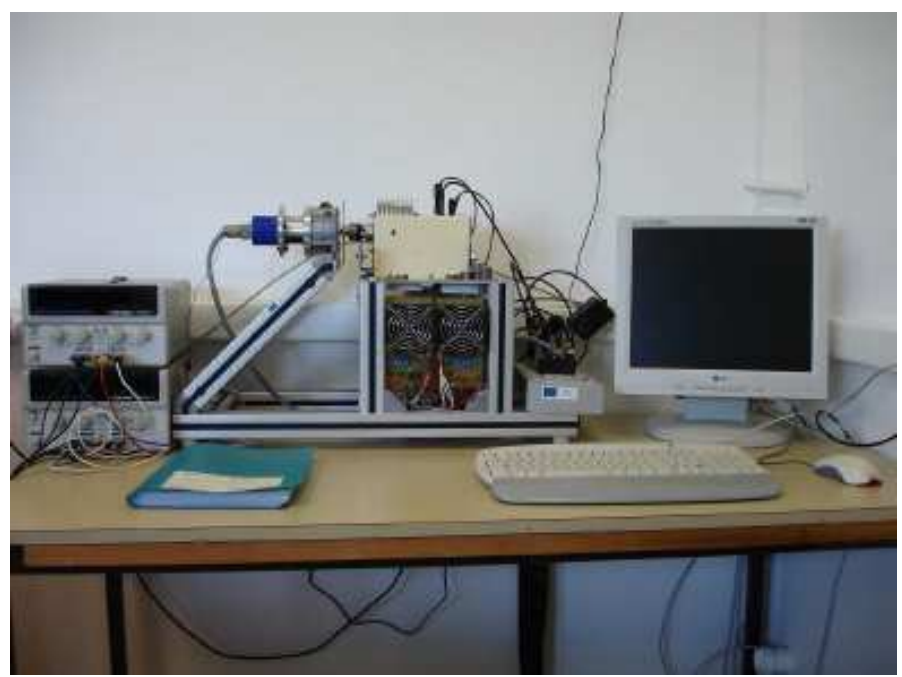

Figure 3. Stepper-motor test bench.

The experimentations are realized using a stepper motor bench developed in LAGIS at École Centrale de Lille (see Fig. 3). The motor parameters have been identified using a mechanical sensorless procedure described in (Delpoux et al. 2012). The motor characteristics, with coils in series are

- Inductance $L=9 m H$,

- Resistance $R=3.01 \Omega$,

- Back-EMF constant $K=0.27 N . m / A$,

- Moment of inertia $J=3 \cdot 18 \cdot 10^{-4} \mathrm{~kg} \cdot \mathrm{m}^{2}$,

- Coefficient of viscous friction $f_{v}=2 \cdot 37 \cdot 10^{-3}$ N.m.S $/ \mathrm{rad}$,

- Coefficient of Coulomb friction $C_{r}=0.0752 N . m$,

- Number of pole pairs $n_{p}=50$.

It is important to note that number of pole pairs is higher than conventional two-phase permanent magnet synchronous motors. Such a rotor design has a low moment of inertia, resulting in outstanding acceleration behavior. However, this large number of poles produces currents and voltages in the fix frame at higher frequencies, which vary at $n_{p}=50$ times the frequency of the motor rotation. The computer hardware on the test-bench is a dSpace 1104, with ControlDesk software as interface. Using the library RTlib developed by dSpace, the algorithms are implemented in $\mathrm{C}$ language. The input voltages $v_{a}$ and $v_{b}$ of each coils are delivered by two $\mathrm{D} / \mathrm{A}$ outputs of the dSpace and amplified by two linear amplifiers. The currents $i_{a}$ and $i_{b}$ are measured using hall effect sensors with a precision of $1 \%$ of the nominal current $I_{n}=3 \mathrm{~A}$. The power supply provides a maximum voltage $v_{\max }=30 \mathrm{~V}$ and $i_{\max }=3 \mathrm{~A}$. Fig. 2 , show the whole control scheme. The sampling frequency for the experiments is constant and equal to $10^{-4} \mathrm{~s}$.

\subsection{Experimental results}

The performances of the approach are demonstrated by choosing a particular reference trajectory. The objective is to show that the motor is able, starting from a null velocity, to track the reference 
trajectory even for negative velocities. As mentioned before, the open loop control is used for velocities lower than $\left|\omega_{l i m}\right|$, where $\omega_{\text {lim }}$ is chosen equal to $3 \mathrm{rad} . \mathrm{s}^{-1}$. The switching instants are represented in the following figures by a magenta line. The desired trajectory is defined applying (70) twice, the first time starts with a position equal to zero to reach an intermediate position, from 0 to $18 \mathrm{rad}$ in 2 seconds. Then equation (70) is applied from the intermediate position until a final position equal to 0 . This leads to a velocity profile which is positive during the first 2 seconds, and negative the 2 last ones.

\subsubsection{Convergence of the observer based on electrical equations}

The position and the velocity are estimated from the observer (26). In order to be well reconstructed, it must be shown that the observers converge correctly. In Fig. 4 are plotted the currents $i_{f}$ and $i_{g}$ and their estimates $\hat{i}_{f}$ and $\hat{i}_{g}$. The figure shows that the estimated currents are close to the measured ones. The estimations errors $\epsilon_{f}$ and $\epsilon_{g}$ are less that $0.01 \mathrm{~A}$.
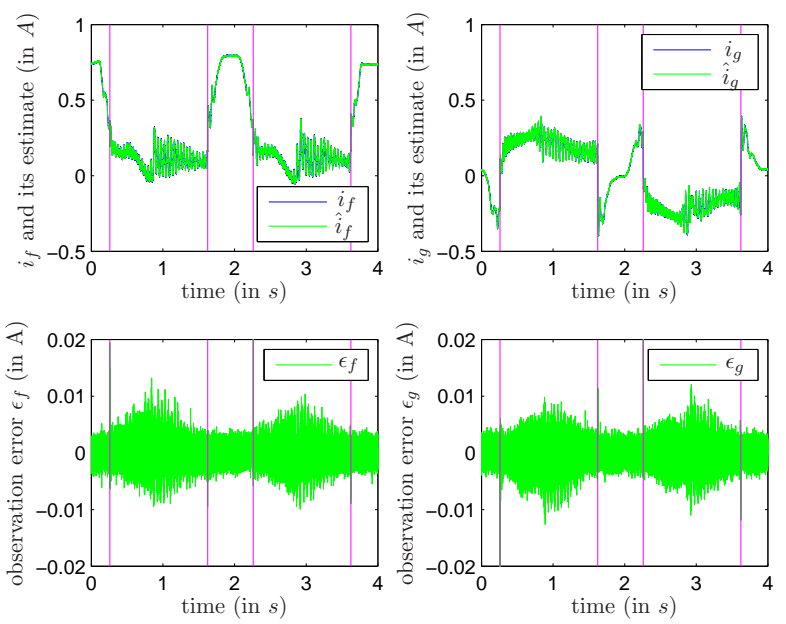

Figure 4. (top) Currents and their estimations, (bottom) observations errors.

The measured disturbance voltages $d_{f}=\frac{K}{L} \omega \sin \left(n_{p} \Delta \theta\right)$ and $d_{g}=-\frac{K}{L} \omega \cos \left(n_{p} \Delta \theta\right)$ are drawn Fig. 5 with their estimates $\hat{d}_{f}$ and $\hat{d}_{g}$ and appear to be closely related. The unknown inputs of the observer are well reconstructed. This shows the convergence of the observer, from these results, one can be able to reconstruct the estimated position and velocity.

\subsubsection{Position and velocity reconstruction}

The position is reconstructed from the estimation of $\cos \left(n_{p} \Delta \theta\right)$ and $\sin \left(n_{p} \Delta \theta\right)$, which enables to estimate the error between the desired trajectory and the motor position. The variable $\Delta \theta_{\text {est }}$ is then computed modulo $\frac{2 \pi}{n_{p}}$. One has to verify that the error between the position and the reference position remains in this interval. In the case where the error leaves the interval, one has to add $\pm 2 \frac{\pi}{n_{p}}$ to compensate for the error. A procedure is implemented to count these "jumps". The integer $k$ is incremented if the estimation leaves the interval by the upper bound and decremented conversely. Such an algorithm is sensitive to measurement noise: a "jump" caused by the noise could be interpreted by the passage to another interval. However, the Super Twisting algorithm plays the role of a filter and provides a continuous estimation $\Delta \theta_{\text {est }}$. From this estimate, the position can be reconstruct as:

$$
\theta_{\text {est }}=\theta_{r}+\Delta \theta_{\text {est }}+2 k \frac{\pi}{N}
$$




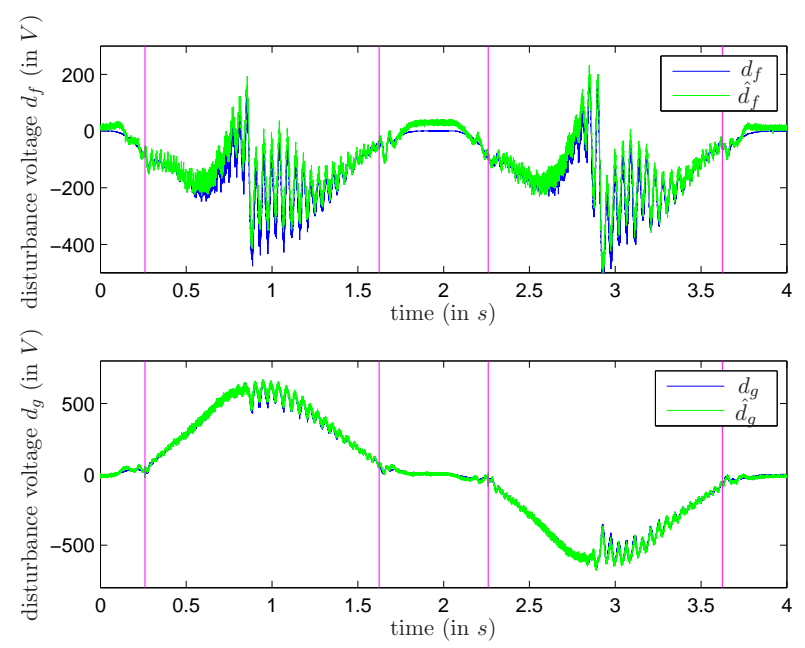

Figure 5. Disturbance voltages and their estimations.

Fig. 6 shows the estimates of these variables, which are plotted while the motor is controlled in closed loop. Firstly it should be noted that the position error is always less than the distance between two poles. The maximum error is around 0.02rad while the angle between two poles is equal to $\frac{2 \pi}{50}=0.126 \mathrm{rad}$. The estimated position is reconstruct using (71) where $k$ is equal to zero. The resulting estimation is plotted Fig. 7. The figure shows the closed loop position tracking. Again the estimated position is plotted when controlled in closed loop. In open loop, the position is equal to the integral of the reference velocity plus the initial position, which is zero at the beginning of the experimentation. Thereafter, the position takes the last estimated value.
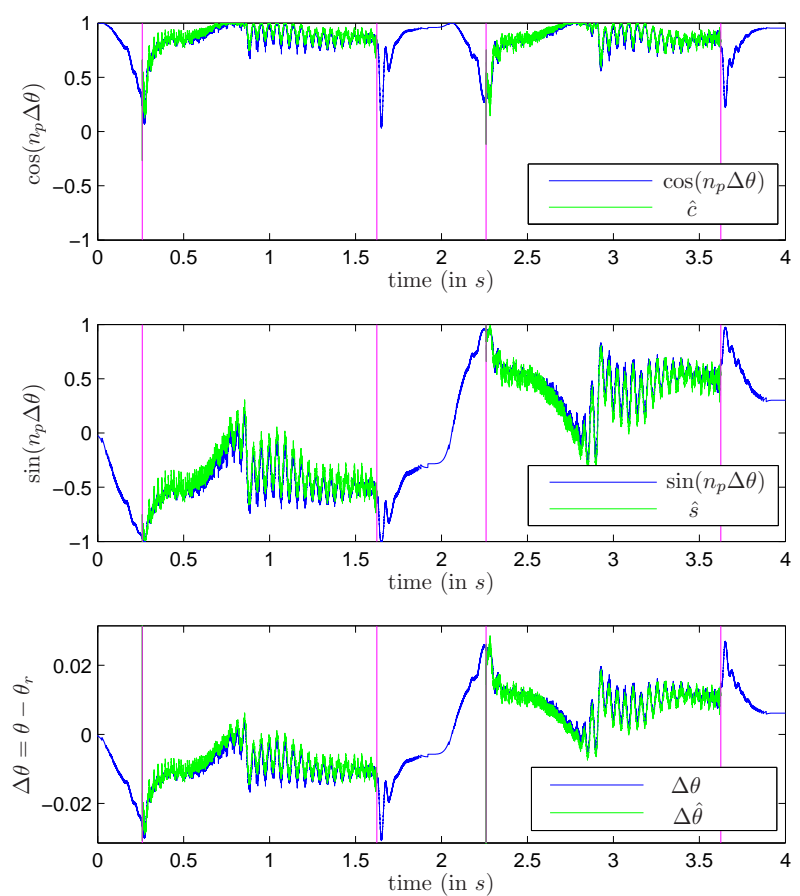

Figure 6. Position error and its estimate. 
The estimated position is plotted in green on the Fig. 7. The second subplot shows the error between the measured and estimated position. The error is less than 0.01rad. The blue curve represents the closed loop performance, and it can be seen that the tracking error in closed loop is less than $0.02 \mathrm{rad}$. This means that over three rounds, the position is tracked without mechanical sensor with a precision less than one degree.
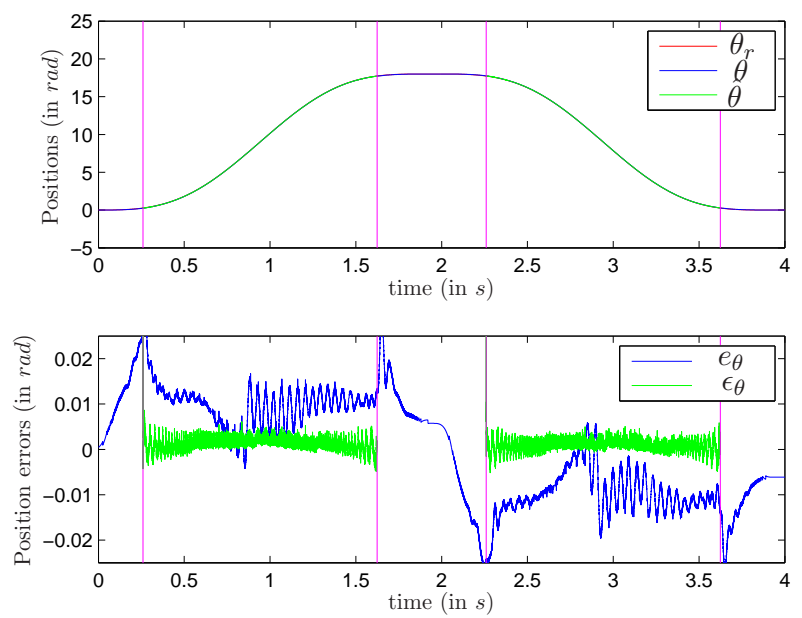

Figure 7. (top) Reference position, measured position and estimated position, (bottom) position tracking error and position observation error.

The velocity estimate is plotted Fig. 8. This figure presents the reference (red), the measured (blue) and the estimated (green) position in the first subplot and in the second subplot the error $\epsilon_{\omega}$ between the measured and the estimated position (green) and the tracking position error $e_{\omega}$. The experiment shows that the motor reaches $20 \mathrm{rad} . \mathrm{s}^{-1}$ with a precision around $1 \mathrm{rad} . \mathrm{s}^{-1}$.

Finally, the acceleration is low pass filtered using a discrete third-order filter (Butterworth). The resulting estimation is plotted with the reference acceleration Fig. 9.
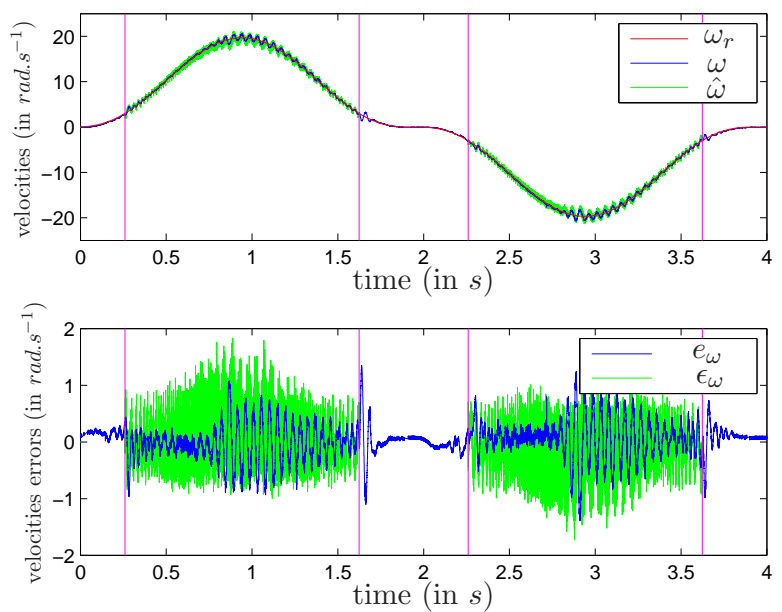

Figure 8. (top) Reference velocity, measured velocity, estimated velocity, (bottom) velocity tracking error, velocity observation error.

Remark 5.1 It is important to recall that the motor used in the experiment has a large number of pole $n_{p}=50$, which limits the speed. In the literature, for example, in (Ortega et al. 2011) $n_{p}=4$, in (Khlaief et al. 2011) $n_{p}=3$. 


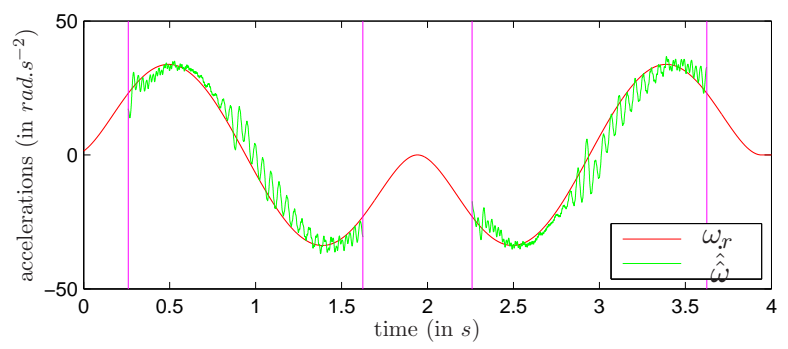

Figure 9. Reference acceleration and observed acceleration.

The results presented in this section prove the effectiveness of the proposed approach.

\section{Conclusion}

There has been considerable interest in developing sensorless control methods for synchronous motors, and permanent magnet stepper motors in particular. The objective is to replace position and velocity sensors with less costly and more reliable current sensors (which are often present anyway).

In this paper, a new approach was proposed for mechanical sensorless control of PMSMs using current sensors only. The model is expressed in a frame, which is obtained from the reference position instead of the measured position. The frame has the advantages of the $(d-q)$ frame without necessity of the measured position and moreover it has the advantage to be valid even when the reference position is different from the measured one. Using a second order sliding mode observer the position, the velocity and the acceleration were estimated. Experimental results that show a very good estimation of this variables. From the observed variables, a trajectory tracking was designed using sliding mode control. This control strategy has the advantage to be robust with respect to external perturbations.

Using an initial scenario, which is used to control the motor when the position is not identifiable, the experimental results show that we are able to realize a position tracking in a large range of velocities, even for negative velocities. The position tracking error is less than 0.02rad which seems to be really reasonable in sensorless applications.

The low speed sensorless control and observability is still an open problem. In many articles, the experimental results are shown at non-null speed only. In this article was described an open loop procedure to overcome this problem. The experimental results have shown very good results with this strategy. However, the theoretical proof is missing. We did not talk neither about the switch between the open loop and the closed loop strategy.

\section{References}

Barbot, J.P., and Floquet, T. (2010), "Iterative higher order sliding mode observer for nonlinear systems with unknown inputs," Dynamics of Continuous, Discrete and Impulsive Systems, 17, 1019-1033.

Bartolini, G., and Pisano, A. (2003), "Global output-feedback tracking and load disturbance rejection for electrically-driven robotic manipulators with uncertain dynamics," International Journal of control, 76, 1201-1213.

Bartolini, G., Pisano, A., Punta, E., and Usai, E. (2003), "A survey of applications of secondorder sliding mode control to mechanical systems," International Journal of Control, 76, 875892.

Bendjedia, M., Ait-Amirat, Y., Walther, B., and Berthon, A. (2012), "Position Control of a Sensorless Stepper Motor," IEEE Transactions on Power Electronics, 27, 578-587. 
Bodson, M., Chiasson, J.N., Novotnak, R.T., and Rekowski, R.B. (1993), "High performance nonlinear feedback control of a permanent magnet stepper motor," IEEE Transactions on Control Systems Technology, 1, 5-14.

Bolognani, S., Zigliotto, M., and Zordan, M. (2001), "Extended-range PMSM sensorless speed drive based on stochastic filtering," IEEE Transactions on Power Electronics, 16, 110-117.

Butt, Q.R., and Bhatti, A.I. (2008), "Estimation of Gasoline-Engine Parameters Using Higher Order Sliding Mode," IEEE Transactions on Industrial Electronics, 55, 3891-3898.

Canale, M., Fagiano, L., Ferrara, A., and Vecchio, C. (2008), "Vehicle Yaw Control via SecondOrder Sliding-Mode Technique," IEEE Control Systems Magazine, 55, 3908-3916.

Chiasson, J.N., and Novotnak, R.T. (1993), "Nonlinear speed observer for the PM stepper motor," IEEE Transactions on Automatic Control, 38, 1584-1588.

Davila, J., Fridman, L., Pisano, A., and Usai, E. (2009), "Finite-time state observation for nonlinear uncertain systems via higher-order sliding modes.," International Journal of Control, $82,1564-1574$.

Defoort, M., Floquet, T., Kökösy, A., and Perruquetti, W. (2008), "Sliding-Mode Formation Control for Cooperative Autonomous Mobile Robots," IEEE Transactions on Industrial Electronics, 55, 3944-3953.

Defoort, M., Nollet, F., Floquet, T., and Perruquetti, W. (2009), "A Third-Order Sliding-Mode Controller for a Stepper Motor," IEEE Transactions on Industrial Electronics, 56, 3337-3346.

Delpoux, R., Bodson, M., and Floquet, T. (2012), "Parameter estimation of permanent magnet stepper motors without position or velocity sensors," in 2012 American Control Conference, Montréal, Québec, pp. 1180-1185.

Drakunov, S.V., Floquet, T., and Perruquetti, W. (2005), "Stabilization and tracking control for an extended Heisenberg system with a drift," Systems $\&$ Control Letters, 54, 435-445.

Emel'yanov, S.V., Korovin, S.V., and Levantovsky, L.V. (1986), "Drift algorithm in control of uncertain processes," Problems of Control and Information Theory, 15, 425 - 438.

Ezzat, M., De Leon, J., Gonzalez, N., and Glumineau, A. (2010), "Observer-controller scheme using high order sliding mode techniques for sensorless speed control of permanent magnet synchronous motor," in 49th IEEE Conference on Decision and Control, Atlanta, Georgia, USA, pp. 4012-4017.

Fiter, C., Floquet, T., and Rudolph, J. (2010), "Sensorless Control of a Stepper Motor Based on Higher Order Sliding Modes," in 8th IFAC Symposium on Nonlinear Control Systems, Bologna, Italy.

Fliess, M., Lévine, J., and Rouchon, P. (1995), "Flatness and defect of nonlinear systems: Introductory theory and examples," International Journal of Control, 61, 1327-1361.

Floquet, T., Barbot, J.P., and Perruquetti, W. (2003), "Higher-order sliding mode stabilization for a class of nonholonomic perturbed systems," Automatica, 39, 1077-1083.

Fridman, L., Davila, J., and Levant, A. (2007), "High-order sliding-mode observation and fault detection," in 46th IEEE Conference on Decision and Control, pp. 4317-4322.

Hamida, M.A., De Leon, J., Glumineau, A., and Boisliveau, R. (2013), "An Adaptive Interconnected Observer for Sensorless Control of PM Synchronous Motors With Online Parameter Identification," IEEE Transactions on Industrial Electronics, 60, 739-748.

Jang, J.H., Ha, J.I., Ohto, M., Ide, K., and Sul, S.K. (2004), "Analysis of permanent-magnet machine for sensorless control based on high-frequency signal injection," IEEE Transactions on Industry Applications, 40, 1595-1604.

Johnson, J.P., Ehsani, M., and Guzelgunler, Y. (1999), "Review of sensorless methods for brushless DC," in 34th IEEE Industry Applications Conference, Vol. 1, Phoenix, AZ, USA, pp. 143 $-150$.

Khlaief, A., Bendjedia, M., Boussak, M., and Gossa, M. (2011), "A Nonlinear Observer for High Performance Sensorless Speed Control of IPMSM Drive," IEEE Transactions on Power Electronics, 27, 3028-3040.

Kim, H., Son, J., and Lee, J. (2011), "A High-Speed Sliding-Mode Observer for the Sensorless 
Speed Control of a PMSM," IEEE Transactions on Industrial Electronics, 58, 4069-4077.

Levant, A. (1993), "Sliding order and sliding accuracy in sliding mode control," International journal of control, 58, 1247-1263.

Levant, A. (2001), "Universal single-input-single-output (SISO) sliding-mode controllers with finite-time convergence," IEEE Transactions on Automatic Control, 46, 1447-1451.

Martinez, R., Alvarez, J., and Orlov, Y. (2008), "Hybrid Sliding-Mode-Based Control of Underactuated Systems With Dry Friction," IEEE Transactions on Industrial Electronics, 55, 3998-4003.

Morimoto, S., Kawamoto, K., Sanada, M., and Takeda, Y. (2002), "Sensorless control strategy for salient-pole PMSM based on extended EMF in rotating reference frame," IEEE Transactions on Industry Applications, 38, 1054-1061.

Morimoto, S., Sanada, M., and Takeda, Y. (2006), "Mechanical Sensorless Drives of IPMSM With Online Parameter Identification," IEEE Transactions on Industry Applications, 42, 1241-1248.

Nollet, F., Floquet, T., and Perruquetti, W. (2008), "Observer-based second order sliding mode control laws for stepper motors," Control Engineering Practice, 16, 429-443.

Orlov, Y., Discontinuous Systems, Springer-Verlag (2009).

Ortega, R., Praly, L., Astolfi, A., Lee, J., and Nam, K. (2011), "Estimation of Rotor Position and Speed of Permanent Magnet Synchronous Motors With Guaranteed Stability," IEEE Transactions on Control Systems Technology, 19, 601-614.

Park, R.H. (1929), "Two-reaction theory of synchronous machines generalized method of analysis-part I," Transactions of the American Institute of Electrical Engineers, 48, 716-727.

Perruquetti, W., and Barbot, J.P., Sliding mode control in engineering, New York: Control Engineering Series, Marcel Dekker Inc (2002).

Pisano, A., Davila, A., Fridman, L., and Usai, E. (2008), "Cascade control of PM-DC drives via second-order sliding mode technique," in 10th International Workshop on Variable Structure Systems, Antalya, Turkey, pp. 268-273.

Riachy, S., Orlov, Y., Floquet, T., Santiesteban, R., and Richard, J.P. (2008), "Second order sliding mode control of underactuated Mechanical systems I: Local stabilization with application to an inverted pendulum," International Journal of Robust and Nonlinear Control, 18, $529-543$.

Schroedl, M. (2004), "Sensorless control of permanent-magnet synchronous machines: An overview," in 11th International Conference on Power Electronics and Motion Control, Riga, Latvia.

Shah, D., Espinosa-Pérez, G., Ortega, R., and Hilairet, M. (2011), "Sensorless Speed Control of Nonsalient Permanent Magnet Synchronous Motors," in 18th IFAC World Congress, Milano, Italy.

Shen, Y., and Huang, Y. (2009), "Uniformly Observable and Globally Lipschitzian Nonlinear Systems Admit Global Finite-Time Observers," IEEE Transactions on Automatic Control, $54,2621-2625$.

Shi, Y., Sun, K., Huang, L., and Li, Y. (2012), "Online Identification of Permanent Magnet Flux Based on Extended Kalman Filter for IPMSM Drive With Position Sensorless Control," Industrial Electronics, IEEE Transactions on, 59, 4169-4178.

Sira-Ramírez, H. (2000), "A Passivity plus Flatness Controller for Permanent Magnet Stepper Motor," Asian Journal of Control, 2, 1-9.

Son, Y.C., Bae, B.H., and Sul, S.K. (2002), "Sensorless operation of permanent magnet motor using direct voltage sensing circuit," in 37th IEEE Industry Applications Conference, Vol. 3, Pittsburgh, Pennsylvania USA, pp. $1674-1678$.

Tomei, P., and Verrelli, C.M. (2011), "Observer-Based Speed Tracking Control for Sensorless Permanent Magnet Synchronous Motors With Unknown Load Torque," IEEE Transactions on Automatic Control, 56, 1484-1488.

Verl, A., and Bodson, M. (1998), "Torque Maximization for Permanent Magnet Synchronous Motors," IEEE Transactions on Control Systems Technology, 6, 740-745. 
Zheng, Z., Li, Y., and Fadel, M. (2007), "Sensorless control of PMSM based on extended kalman filter," in 12th European Conference on Power Electronics and Applications, Aalborg, Denmark, pp. 1-8.

Zhu, H., Xiao, X., and Li, Y. (2009), "A simplified high frequency injection method for PMSM sensorless control," in 6th IEEE International Conference on Power Electronics and Motion Control Conference, Wuhan, China, pp. 401-405.

Zribi, M., Sira-Ramírez, H., and Ngai, A. (2001), "Static and dynamic sliding mode control schemes for a permanent magnet stepper motor," International Journal of Control, 74, 103117. 